\title{
Stabilization of Embedded Defects by Plasma Effects
}

\author{
M. Nagasawa ${ }^{1}$ and R. Brandenberger ${ }^{2,3}$ \\ ${ }^{1}$ Department of Information Science, Faculty of Science, Kanagawa University, Kanagawa 259-1293, JAPAN; \\ ${ }^{2}$ Physics Department, Brown University, Providence, RI 02912, USA; \\ ${ }^{3}$ Department of Physics and Astronomy, University of British Columbia, Vancouver, BC V6T 1Z1, CANADA
}

(February 11, 2018)

\begin{abstract}
In models in which some of the scalar field are charged and some uncharged, interactions with a finite temperature plasma will lead to corrections to the effective potential of the charged fields which may stabilize embedded defects made up of uncharged fields. In these models embedded defects, solutions of the field equations which are unstable at zero temperature, may thus be stable in the early Universe, and may then play an important role in cosmology. Two prototypical examples are the pion string in the theory of strong interactions, and the electroweak Z-string in the standard electroweak theory.
\end{abstract}

PACS numbers: $98.80 \mathrm{Cq}$

\section{INTRODUCTION}

Consider a quantum field theory with an n-dimensional vacuum manifold $\mathcal{M}_{n}$. An illustrative example which will be made use of in most of this Letter is a theory of four real scalar fields $\phi_{i}, i=1, . ., 4$, with a zero temperature potential

$$
V(\phi)=\frac{1}{4} \lambda\left(\sum_{i=1}^{4} \phi_{i}^{2}-\eta^{2}\right)^{2}
$$

which has as its vacuum manifold the three-dimensional sphere $\mathcal{M}_{3}=S^{3}=\left\{\left(\phi_{i}\right): \sum_{i=1}^{4} \phi_{i}^{2}=\eta^{2}\right\}$.

By freezing out certain combinations of the original fields we obtain a sub-manifold $\mathcal{M}_{m}, m<n$, of the original vacuum manifold $\mathcal{M}_{n}$. In the above example we can set $\phi_{3}=\phi_{4}=0$ and obtain the one-dimensional submanifold $\mathcal{M}_{1}=S^{1}=\left\{\left(\phi_{i}\right): \sum_{i=1}^{2} \phi_{i}^{2}=\eta^{2}\right\}$

As is well known $\mathbb{Q}$, the topology of the vacuum manifold determines the types of topological defects which the theory admits. If the topology of $\mathcal{M}_{m}$ is such that $\mathrm{k}$-dimensional defects are possible (in four-dimensional space-time the criterion is $\Pi_{2-k}\left(\mathcal{M}_{m}\right) \neq 1$, where $\Pi_{l}$ is the l'th homotopy group), then it is possible to construct configurations of the unconstrained fields which correspond to k-dimensional defects. If these configurations satisfy the equations of motion of the unconstrained theory, we have an embedded defect 24 . In the above example, the theory of $\left(\phi_{1}, \phi_{2}\right)$ and vacuum manifold $\mathcal{M}_{1}$ admits linear defects called cosmic strings. These string solutions with $\phi_{3}=\phi_{4}=0$ satisfy the full set of equations of motion and are hence embedded strings.

Embedded defects are not topologically stable In general, they are not dynamically stable, either 5 . The defect configurations can unwind by exciting the frozen field combinations. In the above example, the embedded string can unwind by escaping into the $\phi_{3}$ and $\phi_{4}$ field dimensions.

If they were stable during a certain period in the early Universe, embedded defects could play an important role in cosmology. The first important example arises in the Standard Model of strong interactions, which below the confinement scale is described by a sigma model analogous to our toy model of Eq. (1), with $\phi_{1}$ denoting the $\sigma$ field and $\phi_{i}, i=2,3,4$ the three pions. This theory admits no stable defects. Since $\Pi_{3}\left(\mathcal{M}_{3}\right) \neq \mathbf{1}$ there are $\Pi_{3}$ textures 6 (these are $k=-1$ dimensional defects in the above notation), but in the absence of a stabilizing Skyrme term $\Pi_{3}$ textures are unstable 1 . However, by setting the charged pion fields $\phi_{3}=\phi_{4}=0$, we can construct embedded strings (pion strings 8 ). A pion string along the $\mathrm{z}$ axis is given by the configuration

$$
\phi(r, \theta)=\eta \rho(r) e^{i \theta}
$$

where $r, \theta$ are the polar coordinates in the x-y plane and $\phi=\phi_{1}+i \phi_{2}$. The profile function $\rho(r)$ satisfies the boundary conditions $\rho(r) \rightarrow 0$ for $r \rightarrow 0$ and $\rho(r) \rightarrow 1$ for $r \rightarrow \infty$. In Ref. [9] it was shown that due to the anomalous coupling with electromagnetism, pion strings may provide a mechanism for generating primordial galactic magnetic fields.

A second application of stabilized embedded defects is to baryogenesis. Since defects trap energy in the unbroken state and since they are out-of-equilibrium field configurations, they can play an important role in baryogenesis 10 . Defect-mediated baryogenesis may be implemented at the GUT scale 10, at the electroweak scale 11. [2], and possibly even at the QCD scale [3. Embedded defects can contribute to baryogenesis in the same way 11 as topological defects. Consider, for example, the standard electroweak theory based on the gauge group $S U(2) \times U(1)$ with a complex Higgs doublet $\Phi=\left(\phi_{+}, \phi_{0}\right)$, where the subscript indicates the electric charge. The electroweak Z string $[3.15$ is a solution of the equations of motion obtained by embedding the Nielsen-Olesen $U(1)$ string in the following way:

$$
\Phi=\rho_{N O}(r) e^{i \theta}(0,1), \quad Z_{\mu}=A_{\mu, N O},
$$

where $\rho_{N O}(r)$ and $A_{\mu, N O}$ are the scalar profile function and the gauge field, respectively, of the Nielsen-Olesen 
string, and $Z_{\mu}$ is the gauge field associated with the (electrically uncharged) $\mathrm{Z}$ boson. It can be shown that for realistic values of the weak mixing angle, the electroweak string is unstable at zero temperature. If stable, electroweak strings could mediate electroweak scale baryon number violating processes 11 .

A third application of embedded defects is their role in mediating interactions between stable topological defects. In Ref. [16] it has been shown that in a $O(3)$ linear sigma model there exists an attractive force between monopoles and embedded walls and that upon contact the monopole charge spreads out on the wall. Thus, embedded walls can mediate a long range force between monopoles (they sweep up monopoles and antimonopoles) and thus alleviate the monopole problem, as proposed in the context of topological walls in Ref. [17]. The collapse of stable embedded defects could also possibly lead to signatures in the X-ray and cosmic ray backgrounds.

There are many possible embedded defects - embedded monopoles the, embedded strings like the abovementioned pion and electroweak strings, and embedded walls 29 . In general, the criteria for the existence of embedded defects are more complicated than in our simple toy model. However, for the rest of this Letter we shall stick to the simple model.

The formation of non-topological defects was studied in [20] and [21]. As shown in [20], the formation probability of electroweak strings is negligibly small if finite temperature effects are neglected. In contrast, if embedded defects were stable at temperatures below the symmetry breaking phase transition, they would be produced in comparable abundance to that of topological defects of the same dimension, by the usual Kibble argument 16 .

As has been pointed out, it is possible thatembedded defects can be stabilized by bound states 22.23. or by external electric 24 and magnetic fields 25 . These mechanisms, however, are not generic. In this Letter we propose a new and rather generic stabilization mechanism which works whenever the fields excited in the embedded defects are uncharged and the non-excited fields are charged, and when the system is in a finite temperature plasma made up of charged fields. In our examples, the charge will be the usual electric charge. The mechanism, however, is more general and applicable to any kind of charge, provided that the plasma is made up of particles which carry that charge.

The basic idea is as follows: interactions with the charged plasma will generate corrections to the effective potential for the scalar fields which lift the potential in the directions of the charged fields. This reduces the vacuum manifold $\mathcal{M}_{n}$ of the zero temperature theory to a lower dimensional sub-manifold $\mathcal{M}_{m}$, thus providing a way to stabilize embedded defects of the full theory which are topological defects from the point of view of $\mathcal{M}_{m}$.

Our effect is different from an ordinary finite temperature effect. We assume that the scalar fields are not in thermal equilibrium with the plasma, but that the gauge fields which carry the charge force are, in contrast to the usual framework of finite temperature field theory where it is assumed that all fields are in thermal equilibrium. It has been shown 23. that ordinary finite temperature effects (which lift the effective potential in all field directions) cannot in general stabilize embedded defects.

\section{ANALYTICAL CONSIDERATIONS}

As a toy model for the analytical study of the stabilization of embedded defects by plasma effects we consider the chiral limit of the QCD linear sigma model, involving the sigma field $\sigma$ and the three pions $\vec{\pi}=\left(\pi^{0}, \pi^{1}, \pi^{2}\right)$, given by the Lagrangian

$$
\mathcal{L}_{0}=\frac{1}{2} \partial_{\mu} \sigma \partial^{\mu} \sigma+\frac{1}{2} \partial_{\mu} \vec{\pi} \partial^{\mu} \vec{\pi}-\frac{\lambda}{4}\left(\sigma^{2}+\vec{\pi}^{2}-v^{2}\right)^{2},
$$

where $v^{2}$ is the ground state expectation value of $\sigma^{2}+\vec{\pi}^{2}$. In the following, we will denote the potential in (14) by $V_{0}$.

Two of the scalar fields, the $\sigma$ and $\pi_{0}$, are electrically neutral, the other two are charged. Introducing the coupling to electromagnetism, it is convenient to write the scalar field sector $\mathcal{L}$ of the resulting Lagrangian in terms of the complex scalar fields

$$
\pi^{+}=\frac{1}{\sqrt{2}}\left(\pi^{1}+i \pi^{2}\right), \pi^{-}=\frac{1}{\sqrt{2}}\left(\pi^{1}-i \pi^{2}\right) .
$$

According to the minimal coupling prescription we obtain

$$
\mathcal{L}=\frac{1}{2} \partial_{\mu} \sigma \partial^{\mu} \sigma+\frac{1}{2} \partial_{\mu} \pi^{0} \partial^{\mu} \pi^{0}+D_{\mu}^{+} \pi^{+} D^{\mu-} \pi^{-}-V_{0},
$$

where

$$
D_{\mu}^{+}=\partial_{\mu}+e A_{\mu}, \quad D_{\mu}^{-}=\partial_{\mu}-e A_{\mu} .
$$

If the gauge fields are part of the finite temperature plasma, we can insert (7) into (6) and do a Hartree-like approximation by substituting

$$
<A_{\mu}>=0, \quad<A_{\mu} A^{\mu}>=\kappa T^{2}
$$

where $T$ is the temperature and $\kappa$ is a numerical constant of order unity, to extract from (6) an effective potential for the scalar fields of the form

$$
V_{T, e f f}(\sigma, \vec{\pi})=V_{0}+\frac{1}{2} e^{2} \kappa T^{2}\left(\left(\pi^{1}\right)^{2}+\left(\pi^{2}\right)^{2}\right) .
$$

At zero temperature, the vacuum manifold is $S^{3}$, but at finite temperature it reduces to $S^{1}$. Thus, it is not unreasonable to suspect that at high $T$ the embedded string (2) is stabilized.

To check the stability of the pion string (2), we consider the following variational ansatz for an unstable mode 
which obeys the cylindrical symmetry of (2) but escapes into the charged field directions:

$$
\begin{aligned}
\phi=\sigma+i \pi^{0} & =v \rho(r) e^{i \theta} \rho(r)=\left(1-e^{-\mu r}\right) \\
\pi^{1}(r) & =v \chi(r),
\end{aligned}
$$

where $\mu$ is the width of the string (note that since the temperature only affects the charged fields and the embedded defects is made up of neutral field, the width will be independent of $T$ ) and $\chi \ll 1$. We now calculate the mass per unit length $I$ of the configuration (10) and compare it with the corresponding value $I_{0}$ for the embedded string $(\chi=0)$. We obtain

$$
I-I_{0}=\pi v^{2} \int_{0}^{R} d r r \chi^{2}\left[e^{2} \kappa T^{2}-2 \lambda v^{2} e^{-\mu r}\left(1-\frac{1}{2} e^{-\mu r}\right)\right] .
$$

The condition for stability is $I-I_{0}>0$ for all $\chi(r)$. As a sufficient condition we get

$$
T>2 \lambda^{1 / 2} \kappa^{-1 / 2} e^{-1} v=T_{D} .
$$

If this temperature $T_{D}$ is smaller than the temperature $T_{c}$ of the phase transition, then there will be a period $T \in\left[T_{D}, T_{c}\right]$ during which embedded defects are stable. The critical temperature $T_{c}$ is ehtained from the finite temperature effective potential 26. Up to factors of order unity we obtain in our toy model $T_{c} \simeq v$. Hence, provided that $\lambda e^{-2} \ll 1$ there is a period in the early Universe during which embedded defects are stable.

So far, however, we have only studied the stability towards perturbations maintaining the cylindrical symmetry. It is also important to analyze the stability towards fragmentation. To do this, we can consider the variational ansatz (10) with a z-dependence of $\rho$ and $\chi$. The only way this changes the previous analysis is by adding to $I$ extra positive definite terms coming from spatial gradient energies from z-derivatives. Hence, it is more difficult to create local z-dependent perturbations than z-independent ones. Thus, an embedded string with $T<T_{D}$ is also stable towards fragmentation.

\section{NUMERICAL RESULTS}

We have simulated the formation of embedded defects in the presence of a charged plasma using a code based on the one employed in [27]. Four scalar fields $\phi$ are evolved numerically on a three-dimensional lattice by means of the equations of motion derived from (14) with the potential $V_{0}$ replaced by the effective potential (9). All dimensional quantities were rescaled by appropriate powers of $v$ to make them dimensionless. For most simulations, a box size of $50^{3}$ was used (we checked that the basic results were insensitive to the box size by executing $100^{3}$ and $200^{3}$ box simulations.). The spatial resolution was $\Delta x=v^{-1}$, and the time steps were chosen as

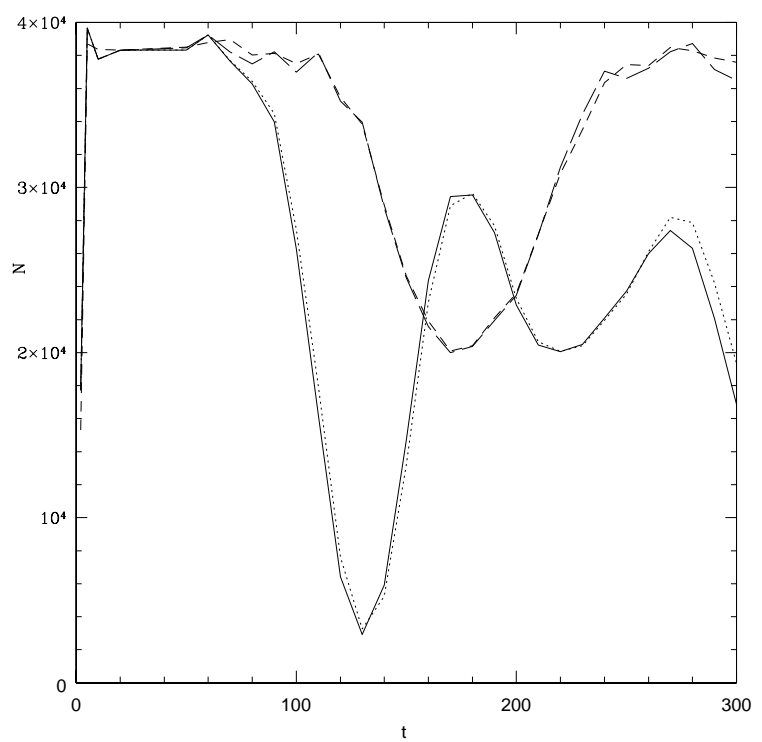

FIG. 1. Time evolution of the number $N$ of points where $|\phi|<0.5 v$. The simulations start at $t=1$ and the result is the averaged value over 20 simulations for different initial phase configuration in a $50^{3}$ box. The solid line corresponds to $T=10^{-3} v$, the dotted one to $T=10^{-2} v$, the long-dashed one to $T=10^{-1} v$, and the short-dashed one to $T=v$.

$\Delta t=\frac{1}{100} \Delta x$. We used two different sets of initial conditions. The results shown below are for true vacuum initial conditions, choosing the fields to be randomly distributed (on a length scale of $\Delta x$ ) over the entire vacuum manifold $\mathcal{M}_{n}=S^{3}:|\phi(x)|=v, \dot{\phi}(x)=0$. We also performed simulations with false vacuum initial conditions determined by $\phi(x)=0,|\dot{\phi}(x)|=T^{2}$, with random orientations of the direction of $\dot{\phi}$ in the field tangent space.

The presence of embedded defects implies the existence of points in space with $|\phi(x)|<\alpha v$, where $\alpha<1$ is an arbitrary constant which determines what is meant by the core radius of the defect (and chosen to be $\alpha=1 / 2$ in Figs. 1 and 2). For true vacuum initial conditions, we expect that the number $N$ of grid points which satisfy the above criterion is initially independent of the temperature $T$ (and simply indicative of the random fluctuations set up by the initial gradient energy). As a function of time, we expect the number of points to decrease if defects are unstable, but to converge to a constant value if defects are stable. Thus, we expect an abrupt increase in $N$ at later times at the stabilization temperature $T_{D}$. We observed this in our simulations. Fig. 1 shows the results for the case $\kappa=e=1$ and $\lambda=10^{-2}$. The numerically determined value of $T_{D}$ lies between $10^{-2} v$ and $10^{-1} v$, in good agreement with the analytical upper bound of (12). The fact that there are points with $|\phi(x)|<\alpha v$ for all temperatures is due to the large energy in the system 

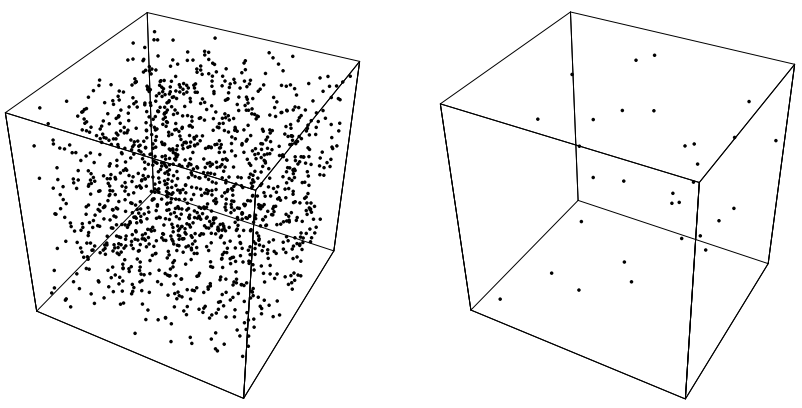

FIG. 2. The three-dimensional distribution of cells where $|\phi|<\alpha v$ at $t=80$ for simulations including the damping term. The left graph is for $T=v, \alpha=0.1$ and the right one for $T=10^{-3} v, \alpha=0.15$.

due to the initial gradient energy which leads to fluctuations. In an expanding Universe, these fluctuations would be damped. However, expansion is not included in our code.

We can introduce a damping term in order to reduce the effects of fluctuations. In Fig. 2 we compare the three-dimensional distribution of points with $|\phi(x)|<\alpha v$ in a simulation with $T=v$ (stable defects expected) and with $T=10^{-3} v$ (no stable defects expected). In the first case, although the zero point constraint is more stringent than the second one due to the smaller alpha, strings of grid points with $|\phi(x)|<\alpha v$ are apparent, whereas such strings are absent in the second case.

\section{DISCUSSION}

We have discussed the stabilization of embedded defects made up of neutral fields in the presence of a charged plasma. We illustrated the effect in the context of pion strings which can be stabilized by the electromagnetic plasma. However, the same mechanism applies to other charges, for example to the color-charged plasma above the QCD confinement scale which has the potential of stabilizing color-neutral embedded defects.

The stabilization of neutral embedded defects is due to finite temperature plasma effects which add (to leading order) quadratic corrections to the effective potential for the charged fields. No external charged fields (as in the stabilization mechanism of [24] in which embedded defects which couple to the external charge are stabilized by an induced angular momentum) are required.

For our mechanism to operate it is important that the fields which make up the embedded defects are no longer in thermal equilibrium whereas the gauge fields which carry the charges are still thermally excited. This condition is easier to realize for global defects such as pion strings than for local defects such as electroweak strings in which the gauge fields themselves play an important role.

Important questions for future study are the formation probability and length distribution of stabilized embedded strings. Unlike topologically stable strings, stabilized embedded strings may have ends (in which case they are expected to be short-lived). Answers to these questions will be crucial in order to be able to discuss the cosmological consequences of the scenario. Work on this topic is in progress.

\section{Acknowledgments}

MN acknowledges the kind hospitality of Brown University and University of British Columbia (UBC) where most of this work was done. R.B. thanks W. Unruh for hospitality at UBC. We are grateful to T. Vachaspati for comments on a draft of the paper. The work of R.B. is supported in part by the U.S. Department of Energy under Contract DE-FG02-91ER40688, TASK A.

[1] T.W.B. Kibble, J. Phys. A9, 1387 (1976).

[2] T. Vachaspati and M. Barriola, Phys. Rev. Lett. 69, 1867 (1992).

[3] T. Vachaspati, Phys. Rev. Lett. 68, 1977 (1992); Nucl. Phys. B397, 648 (1993).

[4] M. Barriola, T. Vachaspati and M. Bucher, Phys. Rev. D50, 2819 (1994).

[5] M. James, L. Perivolaropoulos and T. Vachaspati, Phys. Rev. D46, 5232 (1992); Nucl. Phys. B395, 534 (1993); M. Goodband and M. Hindmarsh, Phys. Lett. B363, 58 (1995).

[6] R. Davis, Phys. Rev. D35, 3705 (1987).

[7] N. Turok, Phys. Rev. Lett. 63, 2625 (1990).

[8] X. Zhang, T. Huang and R. Brandenberger, Phys. Rev. D58, 027702 (1998), hep-ph/9711452.

[9] R. Brandenberger and X. Zhang, Phys. Rev. D, in press (1999), hep-ph/9808306.

[10] R. Brandenberger, A.-C. Davis and M. Hindmarsh, Phys. Lett. B263, 239 (1991).

[11] R. Brandenberger and A.-C. Davis, Phys. Lett. B308, 79 (1993).

[12] R. Brandenberger, A.-C. Davis and M. Trodden, Phys. Lett. B335, 123 (1994); R. Brandenberger, A.-C. Davis, T. Prokopec and M. Trodden, Phys. Rev. D53, 4257 (1996).

[13] R. Brandenberger, I. Halperin and A. Zhitnitsky, hep$\mathrm{ph} / 9808471$.

[14] H. Nielsen and P. Olesen, Nucl. Phys. B61, 45 (1973).

[15] Y. Nambu, Nucl. Phys. B130, 505 (1977); K. Huang and R. Tipton, Phys. Rev. D23, 3050 (1981); N. Manton, Phys. Rev. D28, 2019 (1983).

[16] S. Alexander, R. Brandenberger, R. Easther and A. Sornborger, hep-ph/9903254.

[17] G. Dvali, H. Liu and T. Vachaspati, Phys. Rev. Lett. 80, 2281 (1998). 
[18] R. Brandt and F. Neri, Nucl. Phy. B161, 253 (1979).

[19] C. Bachas and T. Tomaras, Phys. Rev. Lett. 76, 356 (1996).

[20] M. Nagasawa and J. Yokoyama, Phys. Rev. Lett. 77, 2166 (1996).

[21] A. Achucarro, J. Borrill and A. Liddle, hep-ph/9802306.

[22] T. Vachaspati and R. Watkins, Phys. Lett. B318, 163 (1993).

[23] R. Holman, S. Hsu, T. Vachaspati and R. Watkins, Phys. Rev. D46, 5352 (1992).

[24] L. Perivolaropoulos, Phys. Rev. D50, 962 (1994).

[25] J. Garriga and X. Montes, Phys. Rev. Lett. 75, 2268 (1995).

[26] L. Dolan and R. Jackiw, Phys. Rev. D9, 3320 (1974); for a review see e.g. R. Brandenberger, Rev. Mod. Phys. 57, 1 (1985).

[27] M. Nagasawa, K. Sato and J. Yokoyama, Publ. Astro. Soc. of Japan 45, 755 (1993). 